\title{
Radio fiber bursts and fast magnetoacoustic wave trains
}

\author{
M. Karlický ${ }^{1}$, H. Mészárosová ${ }^{1}$, and P. Jelínek ${ }^{2}$ \\ 1 Astronomical Institute of the Academy of Sciences of the Czech Republic, 25165 Ondřejov, Czech Republic \\ e-mail: karlicky@asu.cas.cz \\ 2 University of South Bohemia, Faculty of Science, Institute of Physics and Biophysics, Branišovská 10, 37005 České Budějovice, \\ Czech Republic
}

Received 28 August 2012 / Accepted 28 November 2012

\begin{abstract}
Aims. We present a model for dm-fiber bursts that is based on assuming fast sausage magnetoacoustic wave trains that propagate along a dense vertical filament or current sheet.

Methods. Eight groups of dm-fiber bursts that were observed during solar flares were selected and analyzed by the wavelet analysis method. To model these fiber bursts we built a semi-empirical model. We also did magnetohydrodynamic simulations of a propagation of the magnetoacoustic wave train in a vertical and gravitationally stratified current sheet.

Results. In the wavelet spectra of the fiber bursts computed at different radio frequencies we found the wavelet tadpoles, whose head maxima have the same frequency drift as the drift of fiber bursts. It indicates that the drift of these fiber bursts can be explained by the propagating fast sausage magnetoacoustic wave train. Using new semi-empirical and magnetohydrodynamic models with a simple radio emission model we generated the artificial radio spectra of the fiber bursts, which are similar to the observed ones.
\end{abstract}

Key words. Sun: flares - Sun: corona - Sun: radio radiation - Sun: oscillations

\section{Introduction}

Fiber bursts are considered to be a fine structure of the broadband type IV radio bursts. In the dynamic spectrum, they occur in groups of many single fibers, which are narrowband and have almost the same frequency drift. Because this frequency drift is between those of types II and III bursts, the fiber bursts are also called the intermediate-drift bursts (Bernold \& Treumann 1983; Aurass et al. 1987; Benz \& Mann 1998; Jiřička et al. 2001).

There are two types of models of these bursts: a) one based on the whistler waves (Kuijpers 1975; Chernov 1976; Mann et al. 1987) and b) one based on the Alfvén waves or solitons (Treumann et al. 1990). Both these types of models can be used for estimating the coronal magnetic fields (Benz \& Mann 1998; Rausche et al. 2007), which is otherwise very difficult to determine. For this reason the theory and interpretation of fiber bursts are very important. However, these two types of models give different values for the magnetic field strength, and there is still a debate about which models are correct.

Besides these models, Kuznetsov (2006) proposes a model in which the fiber bursts are generated by a modulation of the radio emission by magnetohydrodynamic waves. He also proposes that these waves could be magnetoacoustic waves of a sausage mode type that propagates along the dense coronal loop.

It is known that the structures with higher plasma density (like dense loops or current sheets) act as waveguides, where the fast magnetoacoustic waves are trapped (Roberts et al. 1984). If these waves are impulsively triggered at some location in such a dense structure, then at some distance from the initiation site along this structure, these propagating waves form the wave trains owing to their dispersion properties. The trains exhibit three phases: (1) periodic phase (long-period spectral components arrive as the first ones at the observation point); (2) quasiperiodic phase (both long- and short-period spectral components arrive and interact); and finally (3) decay phase (Roberts et al. 1983, and 1984). As shown by Nakariakov et al. (2004), wavelet spectra of these trains correspond to the wavelet tadpoles (tail plus head). Detailed numerical simulations of these magnetoacoustic (fast sausage) wave trains and corresponding tadpoles in the density slab, as well as in the current sheet, were performed by Jelínek \& Karlický (2012).

Mészárosová et al. (2009a,b) analyzed several radio events. In the first paper we found the wavelet tadpoles in the gyrosynchrotron radio burst, where all the tadpoles were detected at the same time in the whole frequency range. In second paper, analyzing the 2005 July 11 radio burst (generated by the plasma emission processes), we found the wavelet tadpoles that slowly drifted with the frequency drift corresponding to the drift of the whole group of the fiber bursts. Furthermore, Mészárosová et al. (2011) repeated the wavelet analysis of the 2005 July 11 radio event, considering a much broader range of time periods, as well as longer time intervals than in the previous paper. Thus, we found the tadpoles with the period of $P \approx 1.9 \mathrm{~s}$ and with the fast frequency drift corresponding to the drift of individual fiber bursts. Based on this, we proposed that the fiber bursts are generated by a modulation of the type IV radio emission by the magnetoacoustic wave trains. Karlický et al. (2011) also found the wavelet tadpoles in sources of the narrowband dm-spikes.

In the present paper, we firstly verify the above-mentioned statement about the similarity of the frequency drifts of individual fiber bursts to head maxima of the corresponding tadpoles by the wavelet analysis of more observed groups of fibers. Then we simulate fibers in a newly developed semi-empirical model. Due to limitations of this model we also made a first attempt to simulate the fiber bursts in a full magnetohydrodynamic (MHD) model with the fast sausage magnetoacoustic wave train propagating in a vertical and gravitationally stratified current sheet. 

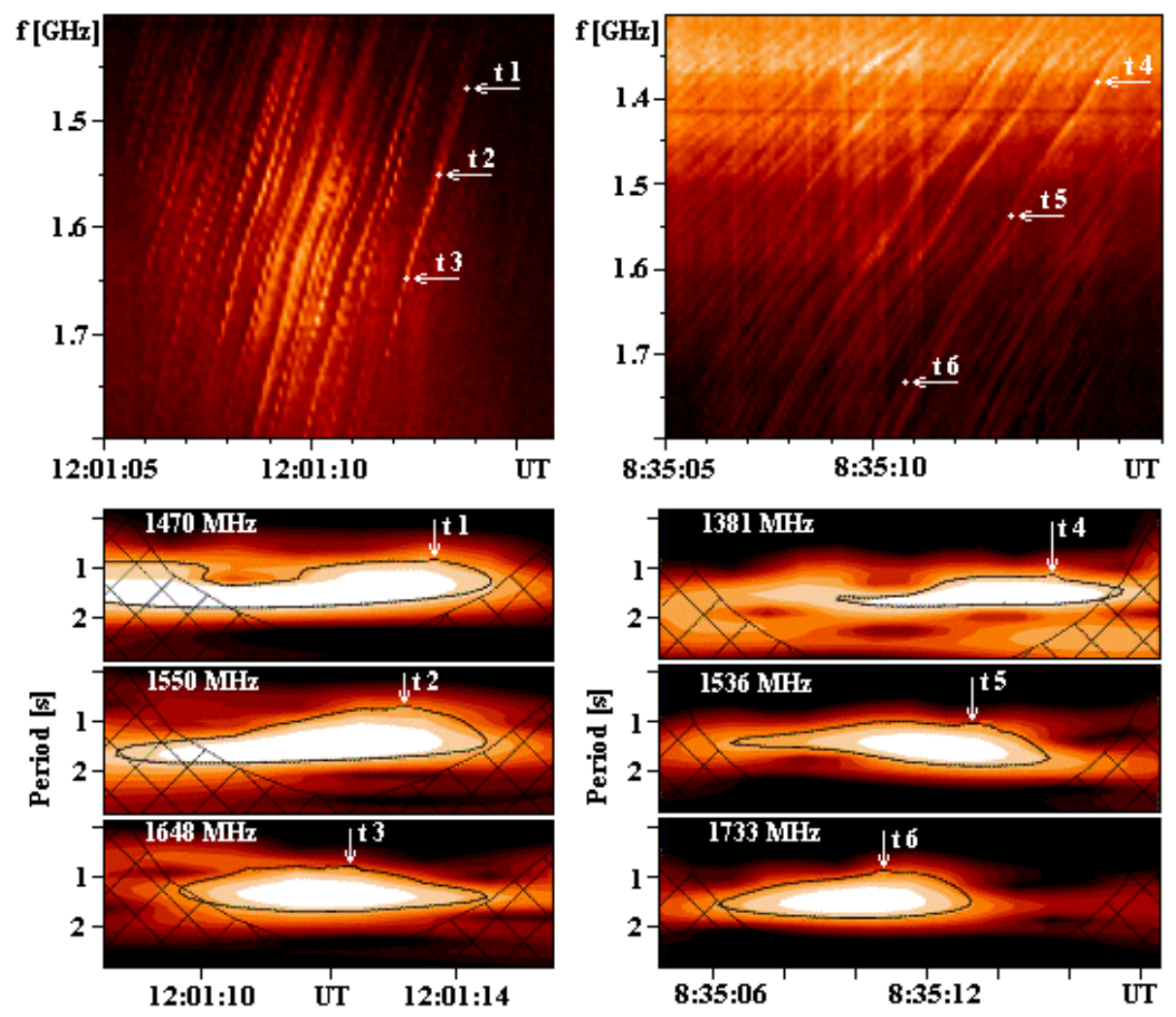

Fig. 1. Upper panels: examples of the fiber bursts at 12:01:05-12:01:16 UT (November 23, 1998, left panel) and 8:35:058:35:17 UT (November 18, 2003, right panel). Bottom part: corresponding wavelet power spectra showing the tadpoles with the period $P=1.4$ s (Table 2 ). In both spectra, at selected frequencies the times of the tadpoles' head maxima were determined $(t 1-t 6)$ and also shown in the upper dynamic radio spectra (upper panels). The tadpole head maxima drift as the fiber bursts.

Using a simple radio emission model we produce artificial radio spectra of the fiber bursts, which are similar to those observed.

The paper is organized as follows: Sect. 2 describes the set of observed fiber bursts and their wavelet analysis. In Sect. 3, we present our semi-empirical model of fiber bursts and their simulations. Section 4 shows a simulation of the fiber bursts using the MHD model. Finally, in Sect. 5 the results are discussed.

\section{Fiber bursts observation and its waletet analysis}

We selected eight decimetric radio events with the fiber bursts recorded during the years 1998-2005 by the Ondřejov radiospectrograph (Jiřička et al. 1993). The times of their observations, the GOES X-ray and $\mathrm{H} \alpha$ characteristics of associated flares are presented in Table 1. Examples of the characteristic radio dynamic spectra that show two typical groups of fiber bursts are presented in upper panels of Fig. 1 (1998 November 23, 12:01:05-12:01:16UT - left panel, and 2003 November 18, 8:35:05-8:35:17UT - right panel). The parameters of all fiber events are summarized in Table 2. The fiber bursts were observed in the frequency range $1000-2000 \mathrm{MHz}$, and their frequency drift $F D_{\mathrm{F}}$ ranges from $-73 \mathrm{MHz} \mathrm{s}^{-1}$ to $-154 \mathrm{MHz} \mathrm{s}^{-1}$.

The selected groups of fibers (Table 1) were analyzed using the wavelet method as described by Mészárosová et al. (2009a,b). We searched for tadpoles in the wavelet power spectra of radio flux time series and selected only dominant tadpoles corresponding to the $99 \%$ confidence level. The confidence level implies a test against a certain background level. If a peak in the wavelet power spectrum is significantly above background spectrum, then it can be assumed to be a true feature with a certain percent of the confidence (Farge 1992). In the present paper the background spectrum is modeled by the red noise, and a computation of the confidence level is performed as described by Torrence \& Compo (1998). The detected wavelet tadpoles and their parameters are summarized in Table 2. Their period
$P$ ranges from 1.1 to $2.3 \mathrm{~s}$, and the drift of their head maxima $F D_{\mathrm{T}}$ is in the range from $-61 \mathrm{MHz} \mathrm{s}^{-1}$ to $-152 \mathrm{MHz} \mathrm{s}^{-1}$. Comparing the frequency drifts of all fiber bursts in Table 2, we can see that the drift of fiber bursts is very similar to that of the head maxima of the corresponding tadpoles (compare values in Cols. 3 and 5 in Table 2). This can be also seen in Fig. 1: see the times $t 1=12: 01: 13.7, t 2=12: 01: 13.1, t 3=12: 01: 12.3$, $t 4=8: 35: 15.5, t 5=8: 35: 13.3, t 6=8: 35: 10.8 \mathrm{UT}$ at the selected frequencies $1470,1550,1648,1381,1536$, and $1733 \mathrm{MHz}$, respectively.

Because the wavelet tadpoles are interpreted as signatures of the fast sausage magnetoacoustic wave trains (e.g. Nakariakov et al. 2004), the similarity of both the drifts indicates that the fiber bursts are physically connected with these magnetoacoustic wave trains. Based on these results we propose that the analyzed dm-fiber bursts are generated by the fast sausage magnetoacoustic wave which modulates the radio emission of superthermal electrons trapped in a flare loop or in current sheet.

\section{Semi-empirical model}

This model is similar to the one presented by Kuznetsov (2006). However, it differs in one aspect: for the fast sausage magnetoacoustic waves considered here, the magnetic and density wave perturbations $\left(b_{z}\right.$ and $\left.\rho_{1}\right)$ are in phase (Erdelyi, priv. comm., 2012), contrary to Kuznetsov (2006), where these perturbations are in anti-phase.

We assume that the magnetoacoustic wave is triggered by a flare and propagates upwards along a dense vertical flare loop. The plasma density and magnetic field in the loop are taken according to the models by Aschwanden $(2002,2004)$, where the magnetic field is expressed as $B(h)=B_{00}\left(1+h / h_{D}\right)^{-3}$ ( $h$ is the height in the solar atmosphere, $h_{D}=75 \mathrm{Mm}$, and $B_{00}$ is the footpoint magnetic field taken in the following runs Nos. 1 and 2 
Table 1. Basic characteristics of the selected events with fiber bursts.

\begin{tabular}{cc|cc|cccc|ccc}
\hline \hline \multirow{2}{*}{ No. } & \multirow{2}{*}{ Flare } & \multicolumn{2}{|c|}{ Radio } & \multicolumn{4}{c|}{ GOES } & \multicolumn{3}{c}{ H $\alpha$} \\
& & Start & End & Start & Max & End & X-ray & Optic. & Position & NOAA \\
& & {$[\mathrm{UT}]$} & {$[\mathrm{UT}]$} & {$[\mathrm{UT}]$} & {$[\mathrm{UT}]$} & {$[\mathrm{UT}]$} & Imp. & Imp. & & AR \# \\
\hline 1 & Nov. 23, 1998a & $11: 51$ & $11: 52$ & $10: 59$ & $11: 21$ & $13: 25$ & M3.1 & $1 \mathrm{~N}$ & S23 E58 & 8392 \\
2 & Nov. 23, 1998b & $11: 58$ & $11: 59$ & $10: 59$ & $11: 21$ & $13: 25$ & M3.1 & $1 \mathrm{~N}$ & S23 E58 & 8392 \\
3 & Nov. 23, 1998c & $12: 00$ & $12: 02$ & $10: 59$ & $11: 21$ & $13: 25$ & M3.1 & 1N & S23 E58 & 8392 \\
4 & Mar. 05, 2000 & $10: 03$ & $10: 04$ & & & & C6.8 & SF & S13 E43 & 8898 \\
5 & Apr. 26, 2001a & $11: 30$ & $11: 31$ & $11: 26$ & $13: 12$ & $13: 19$ & M7.8 & 2B & N17 W31 & 9433 \\
6 & Apr. 26, 2001b & $11: 41$ & $11: 44$ & $11: 26$ & $13: 12$ & $13: 19$ & M7.8 & 2B & N17 W31 & 9433 \\
7 & Nov. 18, 2003 & $08: 34$ & $08: 36$ & $08: 12$ & $08: 31$ & $08: 59$ & M3.9 & 1F & N00 E19 & 10501 \\
8 & Jul. 11, 2005 & $16: 31$ & $16: 43$ & $16: 30$ & $16: 38$ & $16: 50$ & C1.1 & SF & N09 W52 & 10786 \\
\hline
\end{tabular}

Table 2. Parameters of the fiber bursts and associated wavelet tadpoles.

\begin{tabular}{l|cc|cc}
\hline \hline & \multicolumn{2}{|c|}{ Fiber bursts } & \multicolumn{2}{c}{ Wavelet tadpoles } \\
No. & $\begin{array}{c}\text { Frequency range } \\
{[\mathrm{MHz}]}\end{array}$ & $\begin{array}{c}F D_{\mathrm{F}} \\
{\left[\mathrm{MHz} \mathrm{s}^{-1}\right]}\end{array}$ & $\begin{array}{c}\text { Period } \\
{[\mathrm{s}]}\end{array}$ & $\begin{array}{c}F D_{\mathrm{T}} \\
{\left[\mathrm{MHz} \mathrm{s}^{-1}\right]}\end{array}$ \\
\hline 1 & $1456-2000$ & -154 & 1.5 & -152 \\
2 & $1370-1800$ & -130 & 1.5 & -122 \\
3 & $1370-1800$ & -128 & 1.4 & -127 \\
4 & $1000-1330$ & -144 & 1.1 & -136 \\
5 & $1050-1600$ & -92 & 2.0 & -87 \\
6 & $1000-1600$ & -76 & 2.3 & -61 \\
7 & $1100-2000$ & -73 & 1.4 & -75 \\
8 & $1100-1800$ & -78 & 1.9 & -86 \\
\hline
\end{tabular}

as $\left.B_{00}=65 \mathrm{G}\right)$. The plasma density and magnetic field profiles used in the model are shown in Fig. 2.

Although a real wave evolution is complex (see Roberts et al. 1984; Nakariakov et al. 2004, and also the following MHD model), for a simplification we take the velocity of this wave perturbation as the Alfvén speed $v_{\mathrm{A}}$ computed along the loop. The ratios of wave perturbations is taken as $b_{z} / B_{0}=\rho_{1} / \rho_{0} \ll 1$, where $B_{0}$ and $\rho_{0}$ mean the local magnetic field and density in the Aschwanden's models.

We took a wave in the form of the wave packet as

$$
\begin{aligned}
F(h, t)= & F_{0} C_{0} \exp \left[-\left(\frac{h-v_{\mathrm{A}}(h) t-h_{0}}{d_{\mathrm{s}}}\right)^{2}\right] \\
& \times \cos \left[\frac{2 \pi\left(h-v_{\mathrm{A}}(h) t-h_{0}\right)}{d_{\mathrm{L}}}\right] \\
C_{0}= & \exp \left[-\left(\frac{t-t_{\mathrm{Amax}}}{d_{\mathrm{t}}}\right)^{2}\right]
\end{aligned}
$$

where $F(h, t)$ means the density $\rho_{1}$ or magnetic field $b_{z}$ wave perturbation, $F_{0}$ is the wave amplitude, $h$ the height in the loop, $t$ the time, $v_{\mathrm{A}}$ the local Alfvén speed, $h_{0}$ the wave initiation height, $d_{\mathrm{s}}$ the spatial width of the wave packet, and $d_{\mathrm{L}}$ the wavelength. By changing $d_{\mathrm{S}}$ for a fixed $d_{\mathrm{L}}$ we can generate the wave for a single fiber or group of fibers. We also added a factor $C_{0}$ that limits radio emission to our chosen frequency interval $1300-1700 \mathrm{MHz}$ (see the following): $t_{\mathrm{Amax}}$ is thus the time of the maximum wave amplitude, and $d_{t}$ the corresponding characteristic time. In our computations the time $t$ changes from 0 to $5 \mathrm{~s}, t_{\text {Amax }}=2.5 \mathrm{~s}$, $d_{\mathrm{t}}=1.5 \mathrm{~s}$, and $h_{0}=16.9 \mathrm{Mm}$. The other parameters are presented in Table 3. This propagating wave packet is then superimposed on the density and magnetic field profiles presented in Fig. 2.

In agreement with Kuznetsov (2006), we assume that the radio emission is produced at the double (harmonic) upper hybrid frequency as a result of a coalescence of two upper hybrid waves, generated by the loss-cone instability of superthermal electrons.


Fig. 2. Plasma density and magnetic field profiles in dependence on the height in the modeled loop. The horizontal full lines in the density plot mean the plasma densities corresponding to the maximum and minimum of frequencies considered in the radio spectra. The vertical dashed lines limit this density interval.

Table 3. Computation parameters.

\begin{tabular}{ccccc}
\hline \hline Run No. & $\rho_{1} / \rho_{0}$ & $b_{z} / B_{0}$ & $d_{\mathrm{s}}(\mathrm{km})$ & $d_{\mathrm{L}}(\mathrm{km})$ \\
\hline 1 & 0.01 & 0.01 & 200 & 800 \\
2 & 0.01 & 0.01 & 800 & 800 \\
\hline
\end{tabular}

Furthermore, we assume that the radio emission is generated with a constant intensity in the whole interval of considered heights. It means that every unit of the volume along the flaring loop (or current sheet) generates the same radio emission. Thus, the intensity of the radio emission at some specific frequency depends only on the gradients of $n_{\mathrm{e}}$ and $B$ in the radio source; i.e., the wave perturbation modulates the radio emission as proposed by Treumann et al. (1990). Then, the resulting radio 


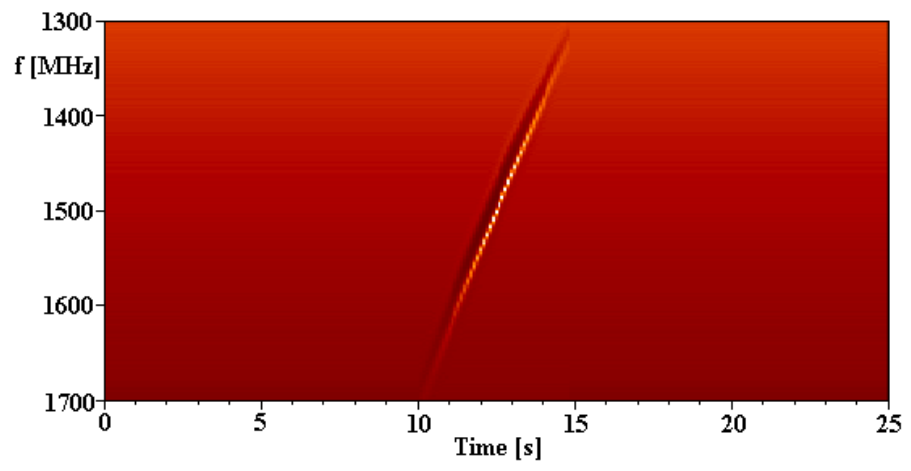

Fig. 3. Computed radio spectrum with the fiber modeled in the semiempirical model for run No. 1.
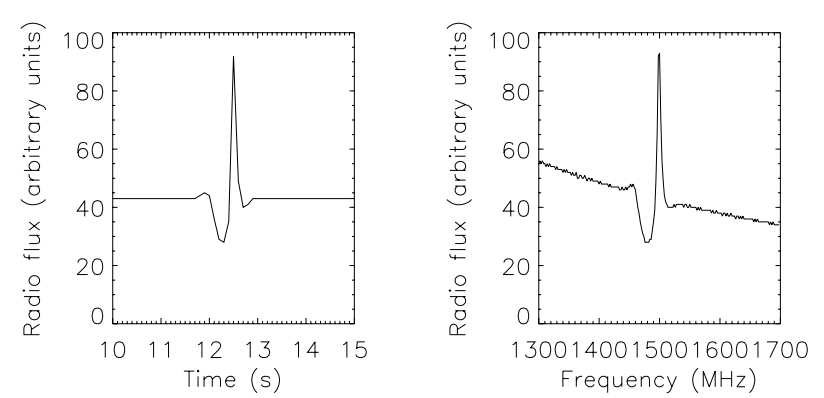

Fig. 4. Example of the time and frequency profiles of the fiber burst modeled in the semi-empirical model for run No. 1. The time profile is plotted at the $1500 \mathrm{MHz}$ frequency and the frequency profile at the time $12.5 \mathrm{~s}$; compare with the spectrum in Fig. 3.

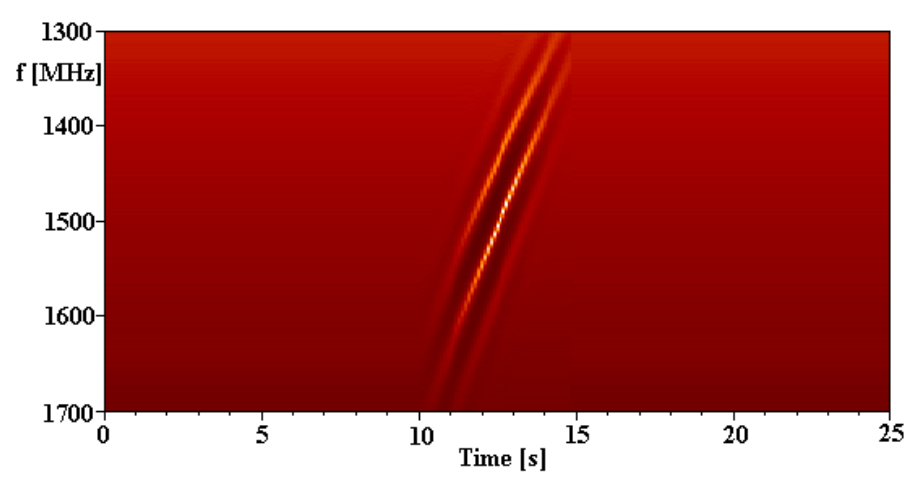

Fig. 5. Computed radio spectrum with the fibers modeled in the semiempirical model for run No. 2.

flux at the specific frequency $f$ can be expressed as a sum of emissions from plasma volumes having the upper hybrid frequency $f_{\text {up }}$ close to $f / 2$.

First, we made two runs with the parameters shown in Table 3. The first run was performed for a single perturbation pulse and the second one for the wave train. The computed radio spectrum is in Fig. 3. (Remark: for better presentation of all computed spectra at their beginning we added the 10 -second radio spectrum of the unperturbed state.) An example of the time and frequency profiles of the resulting fiber for run No. 1 (cuts in the spectrum shown in Fig. 3) is presented in Fig. 4. Besides the emission peak the emission depression on the low-frequency side of the fiber, as observed, is also clearly visible. The radio spectrum and time and frequency profiles for run No. 2, i.e. for the magnetoacoustic wave train, are shown in Figs. 5 and 6. As can be seen here, the wave train generates a series of fiber bursts.
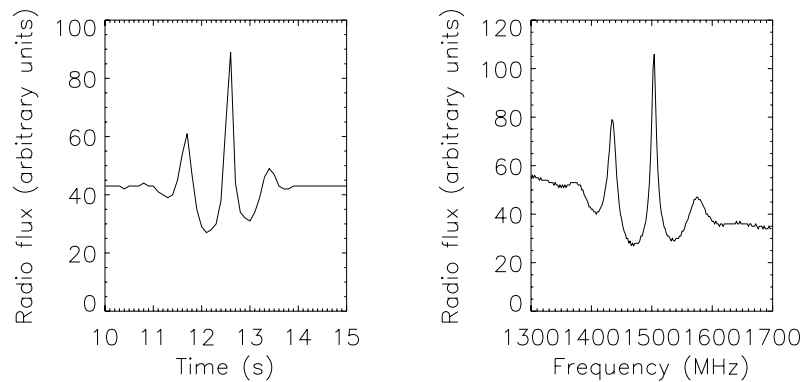

Fig. 6. Example of the time and frequency profiles of the fiber bursts modeled in the semi-empirical model for the run No. 2. The time profile is plotted at $1500 \mathrm{MHz}$ frequency and the frequency profile at time $12.5 \mathrm{~s}$; compare with the spectrum in Fig. 5.

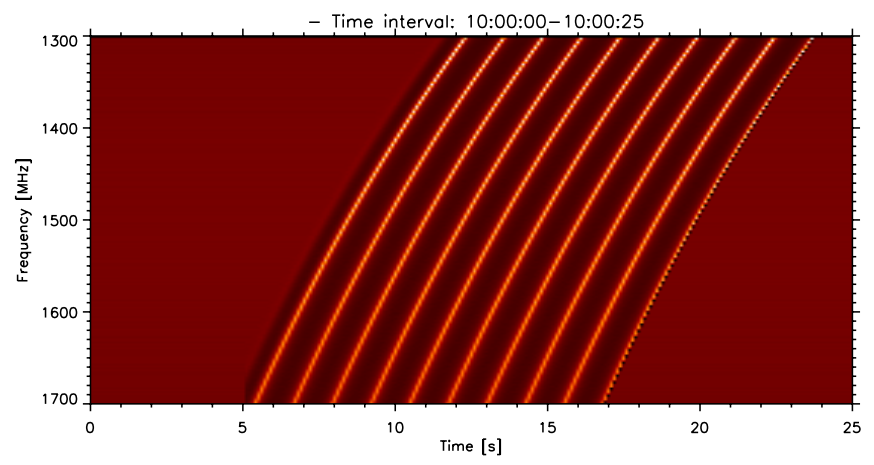

Fig. 7. Computed radio spectrum with the 10 fiber bursts modeled by the semi-empirical model.

Furthermore, as an example, we simulated a group of ten fiber bursts. We consider the wave which is spatially limited to ten wavelengths (wavelength $d_{\mathrm{L}}=800 \mathrm{~km}$ ). It propagates upwards in the solar atmosphere as in runs 1 and 2. However, the magnetic field $B_{00}$ in the used magnetic-field model is $B_{00}=48 \mathrm{G}$. The constant $C_{0}$ (see Eq. (2)) is taken as $C_{0}=1$, which means no global frequency modulation of the radio flux. The computed spectrum with ten fiber bursts is shown in Fig. 7.

Comparing this figure with the radio spectrum in the righthand panel of Fig. 1, we can see some similarities as well as some deviations. Generally, using this semi-empirical model, we can simulate the frequency drift and repetition period of the fiber bursts by varying the Alfvén speed (through the magnetic field $\left.B_{00}\right)$ and wavelength of the magnetoacoustic wave $\left(d_{\mathrm{L}}\right)$. On the other hand, deviations are caused by limited information about conditions in the real radio source and also by limitations in the semi-empirical model used. One of the main problem of this semi-empirical model is that dispersive properties of the magnetoacoustic waves are neglected. Therefore, in the following we describe a first attempt to simulate the fiber bursts in a full MHD model, where these properties are implicitly included.

\section{MHD model}

The main problem of any such model is an initiation of the sufficiently good waveguide for the fast sausage magnetoacoustic waves, which is in equilibrium in the gravitationally stratified solar atmosphere. For a waveguide we can use the dense loop or current sheet, see Jelínek \& Karlický (2012). In an analytical form, the model of the vertical current sheet in the gravitationally stratified atmosphere proposed by Galsgaard \& Roussev (2002) is an appropriate one for initiating the equilibrium waveguide. 
In our case the vertical current sheet (it would be better to call it the current layer) is only a prototype of the waveguide guiding the magnetoacoustic waves. We did not consider any reconnection and fragmentation processes in this current sheet as discussed in Bárta et al. (2011a,b), Karlický et al. (2012), and Cargill et al. (2012), among others. Although the fragmentation is real in these current sheets, we think that such vertical current sheets can exist; see, e.g., the observed current sheet and its width on the order of $1000 \mathrm{~km}$ in Fig. 3 in the paper by Lin et al. (2005).

In our model we use the ideal 2D magnetohydrodynamic (MHD) equations:

$$
\begin{aligned}
\frac{\mathrm{D} \varrho}{\mathrm{D} t} & =-\varrho \nabla \boldsymbol{v}, \\
\varrho \frac{\mathrm{D} \boldsymbol{v}}{\mathrm{D} t} & =-\nabla p+\boldsymbol{j} \times \boldsymbol{B}+\varrho \boldsymbol{g}, \\
\frac{\mathrm{D} \boldsymbol{B}}{\mathrm{D} t} & =(\boldsymbol{B} \cdot \nabla) \boldsymbol{v}, \\
\frac{\mathrm{D} e}{\mathrm{D} t} & =-\gamma e \nabla \cdot \boldsymbol{v}, \\
\nabla \cdot \boldsymbol{B} & =0,
\end{aligned}
$$

where $\mathrm{D} / \mathrm{D} t \equiv \partial / \partial t+\boldsymbol{v} \cdot \nabla$ is the total time derivative, $\varrho$ is a mass density, $\boldsymbol{v}$ flow velocity, $\boldsymbol{B}$ the magnetic field, and $\boldsymbol{g}=\left[0,-g_{\odot}, 0\right]$ is the gravitational acceleration with $g_{\odot}=274 \mathrm{~m} \mathrm{~s}^{-2}$. The current density $\boldsymbol{j}$ in Eq. (4) is expressed as

$\boldsymbol{j}=\frac{1}{\mu_{0}} \nabla \times \boldsymbol{B}$,

where $\mu_{0}=1.26 \times 10^{-6} \mathrm{H} \mathrm{m}^{-1}$ is the magnetic permeability of free space. The specific internal energy $e$ in Eq. (6) is given by

$$
e=\frac{p}{(\gamma-1) \varrho}
$$

with the adiabatic coefficient $\gamma=5 / 3$.

Equations (3)-(6) are solved numerically by the FLASH code (Fryxell et al. 2000; Lee \& Deane 2009). This code implements second- and third-order unsplit Godunov solvers and adaptive mesh refinement (AMR) (see e.g. Chung 2002; Murawski 2002). Spatial resolution of the numerical grid is determined by the AMR method, and we use the AMR grid with a minimum (maximum) level of refinement blocks set to 3 (6). The total number of computational cells covering a whole simulation plane is 267456 . A spatial cell size has to be much smaller than the width of the current sheet in the $x$-direction and the minimal wavelength of the magnetoacoustic waves in the h-direction. In our model the minimal cell sizes are $\Delta x=0.0175 \mathrm{Mm}$ and $\Delta h=0.005 \mathrm{Mm}$ (compared with the half width of the current sheet $w_{\mathrm{CS}}=0.35 \mathrm{Mm}$ and the minimal wavelength of about $1.0 \mathrm{Mm}$ ).

At the start of our numerical calculations we set a refinement procedure in the region covering the current sheet. During the calculations, the FLASH code automatically controlled in each time step the gradient of mass density, which value is then used for refining the grids. For our numerical simulations we used the two-dimensional (2-D) Eulerian box $(-2.25,2.25) \mathrm{Mm} \times$ $(13.0,23.0) \mathrm{Mm}$ in the $x$ - and $h$ - (height) directions, respectively. As a consequence of an extension of the real plasma medium we apply free-boundary conditions at the boundaries of the simulation region, so that the waves can freely leave the simulation box without any significant reflection.
For the description of magnetic field in the vertical current sheet in gravitationally stratified solar atmosphere in the $x-h$ plane we use following expressions:

$$
\begin{aligned}
& B_{x}(x, h)=B_{\mathrm{o}} \frac{w_{\mathrm{cs}}}{\lambda} \ln \left[\cosh \left(\frac{x}{w_{\mathrm{cs}}}\right)\right] \exp \left(-\frac{h}{\lambda}\right), \\
& B_{h}(x, h)=B_{\mathrm{o}} \tanh \left(\frac{x}{w_{\mathrm{cs}}}\right) \exp \left(-\frac{h}{\lambda}\right),
\end{aligned}
$$

where $B_{\mathrm{o}}$ is a magnetic field at $x \rightarrow \infty, w_{\mathrm{CS}}$ is the half width of the current sheet, and $\lambda$ denotes the magnetic scale-height. For an equilibrium $(\boldsymbol{v}=\mathbf{0})$, the Lorentz and gravity forces have to be balanced by the pressure gradient in the entire physical domain

$-\nabla p+\boldsymbol{j} \times \boldsymbol{B}+\varrho \boldsymbol{g}=\mathbf{0}$.

From this condition we can derive the formulae for distribution of the mass density and gas pressure. For the details see Galsgaard \& Roussev (2002) and Jelínek et al. (2012).

In the initial state, we generate the vertical current sheet with the density profile along its axis according to the Aschwanden model (2002). The half width of the current sheet at the height $h=16.9 \mathrm{Mm}$ is taken as $w_{\mathrm{CS}}=0.35 \mathrm{Mm}$. In the paper by Jelínek \& Karlický (2012), we found that the neutral current sheet serves as the same waveguide as the dense slab if the Alfvén speed at the half width of the current sheet is the same as in the slab. Therefore, in the present current sheet we took this Alfvén speed as $690 \mathrm{~km} \mathrm{~s}^{-1}$. The change of this speed in the studied interval of heights in the current sheet is small.

At the start of the numerical simulation, the initial equilibrium state is perturbed by the Gaussian pulse in the $x$-component of velocity and has the following form (e.g. Nakariakov et al. 2004, 2005):

$v_{x}=-A_{0} \frac{x}{\lambda_{h}} \exp \left[-\frac{\left(x-h_{\mathrm{P}}\right)^{2}}{\lambda_{x}^{2}}\right] \exp \left[-\frac{h^{2}}{\lambda_{h}^{2}}\right]$,

where $A_{0}$ is the initial amplitude of the pulse, and $\lambda_{x}=\lambda_{h}=$ $0.35 \mathrm{Mm}$ are the widths of the velocity pulse in the longitudinal and transverse directions. This pulse tends to trigger fast sausage magnetoacoustic waves. The initial perturbation is located at the height $h_{\mathrm{P}}=15 \mathrm{Mm}$.

Using this MHD model, we computed an evolution of the density profiles along the axis of the vertical current sheet (see examples in Fig. 8). These density profiles were then used in computations of the artificial radio spectrum, as well as the time and frequency profiles shown in Figs. 9 and 10. We used the same simple radio emission model as in the case of the semiempirical model. As seen in Fig. 9 the frequency drift of these fiber bursts partly changes. It is caused by the wave dispersive effects (included in this MHD model), which change the density wave profile during the wave propagation. Compare this with the semi-empirical model, where the perturbation profile is rigid. The maximum of fiber bursts are produced at the locations with minimal density gradients and these gradients evolve in time. Similarly, the emission depressions, corresponding to the maximal density gradients, evolve in time.

The detailed analysis of these results shows that the model used for the vertical current-sheet is not the best waveguide for the studied magnetoacoustic waves. The change in the magnetic field gradient across the current sheet is not sharp enough. Therefore, some wave energy escapes from this waveguide. This wave energy leakage limits the wave train length and thus also the number and duration of computed fibers. We hope that in a future model we will generate a better waveguide, in which 


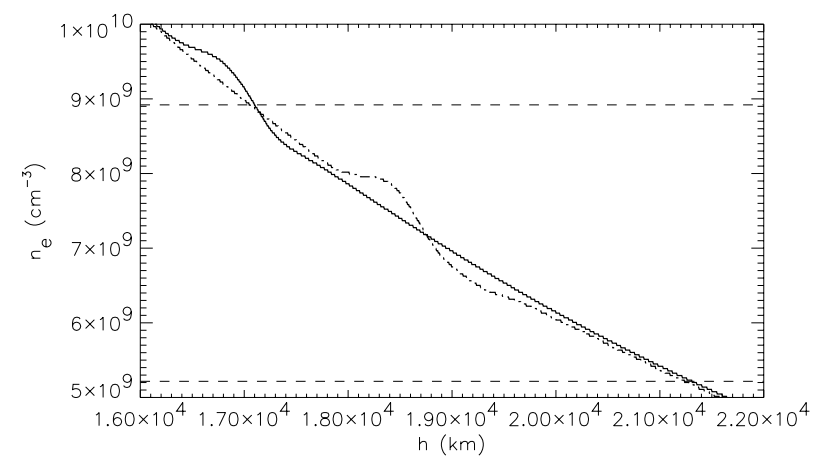

Fig. 8. The density profiles in the MHD model at $11 \mathrm{~s}$ (full line) and $14 \mathrm{~s}$ (dash-dotted line). The times correspond to times in the spectrum in Fig. 9.

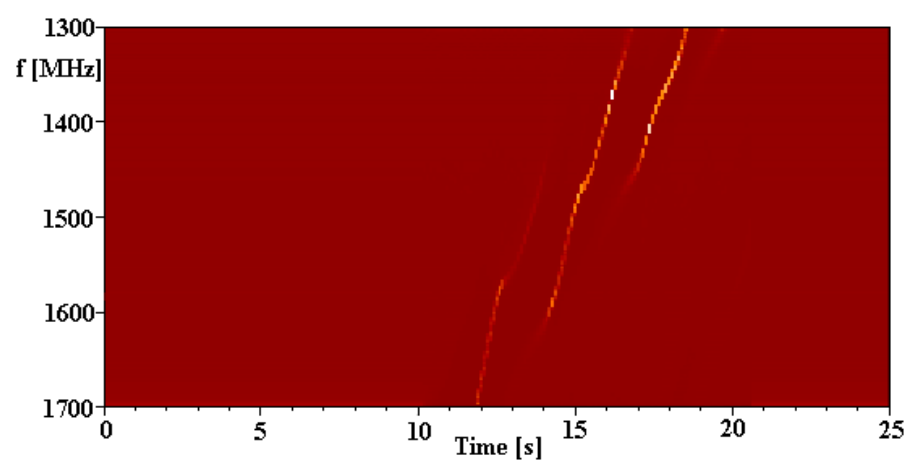

Fig. 9. The computed radio spectrum with the fibers modeled in the MHD model.
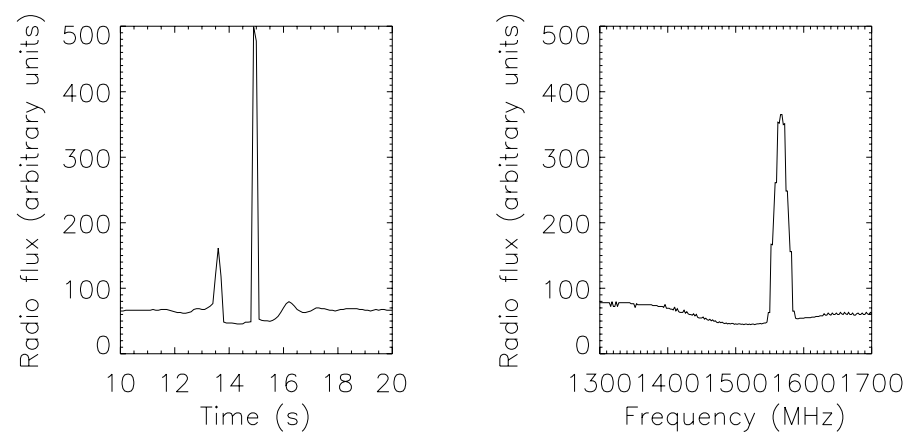

Fig. 10. An example of the time and frequency profiles of the fiber bursts modeled in the MHD model. The time profile is plotted at the $1500 \mathrm{MHz}$ frequency and the frequency profile at the time $14.5 \mathrm{~s}$; compare with the spectrum in Fig. 9.

a longer wave train and thus more fiber bursts will be generated by a single perturbation. On the other hand, more fiber bursts can be generated by a time repetition of the initial perturbation because this is also possible in real conditions. Furthermore, some real radio spectra might show a superposition of the fiber bursts generated in several nearby waveguides.

\section{Discussion and conclusions}

After analyzing eight groups of observed fiber bursts by the wavelet technique, we found a connection between the dmfiber bursts and fast sausage magnetoacoustic waves. Based on this connection, we developed the model of the dm-fiber bursts with the fast sausage magnetoacoustic wave propagating in the waveguide of the enhanced plasma density: (a) in the dense loop according to the Aschwanden's density and magnetic field models (semi-empirical model) and (b) in the vertical and gravitationally stratified current-sheet (MHD) model. In both of these models, using the simple emission model according to Treumann et al. (1990), we computed the artificial radio spectra with the fiber bursts that resembles to the observed ones.

We assumed that every unit of the volume along the flaring loop (or current sheet) generates the same radio emission. The intensity of the radio emission at some specific frequency depends only on the density gradient in the radio source. However, the wave perturbation (with weak local magnetic mirrors) can also modulate the growth rate of the upper hybrid waves through change in the loss-cone angle of superthermal electrons (Winglee \& Dulk 1986; Yasnov \& Karlický 2004) and thus change local emissions. For this purpose, the weighting function $w_{\mathrm{e}}(h(t))$ that expresses these variations can be included in future models.

In all studied decimetric fiber bursts, the frequency drift of the wavelet tadpoles corresponds to the drift of individual fiber bursts. It speaks in favor of the present model with the magnetoacoustic waves in comparison to the model with whistler waves, which is usually considered in the metric frequency range. We propose to do similar wavelet analysis of the metric fiber bursts and to check the validity of their models.

While in the semi-empirical model the form of the wave perturbation is rigid, the MHD model describes perturbations including dispersive properties of the magnetoacoustic waves. It leads to a wavy character of the fiber bursts, which can even be seen in some observed fiber bursts (see e.g. the fibers in the upper-left part of Fig. 1).

The semi-empirical model is very similar to that of Kuznetsov (2006), except that the density and magnetic field perturbations are in phase. The phase relation presented in Kuznetsov (2006) is not correct for the fast sausage magnetoacoustic waves. However, this difference in both the models leads to only small differences in the radio spectrum, because the electron-cyclotron frequency $\omega_{\text {ce }}$ is much lower than the plasma frequency $\omega_{\text {pe }}$, and thus the upper-hybrid frequency roughly equals the plasma frequency and the magnetic field can be neglected in this case. However, the variation of the magnetic field in the magnetoacoustic wave train can influence the growth rate of the upper hybrid waves owing to the loss-cone instability of superthermal electrons. It can modify the radio emission at some locations along the wave train and thus change the fiber burst profiles. This effect is not considered in the present paper.

Comparing both these models, the semi-empirical is easy to use, however, with a limited description of physical processes under study. The MHD model is much more complicated. One of the main problems is to generate the appropriate initial equilibrium state. The MHD model includes the dispersive effects of the propagating magnetoacoustic waves, so it describes the fiber burst generation in a more realistic way. However, the present MHD vertical and gravitationally stratified waveguide (the current sheet according to the Galsgaard \& Roussev model 2002) is not ideal. Owing to its relatively smooth boundaries, the energy of the magnetoacoustic waves escape from this wave guide. The energy of wave decreases, and it is therefore difficult to make long-lasting wave train. This means that we can only simulate a few fiber bursts per one perturbation instead of the long series as in the semi-empirical model. This problem is connected to a solution of the initial equilibrium waveguide. Therefore, to make this MHD model of the fiber bursts more realistic, a better 
initial waveguide needs to be found. On the other hand, the series of fiber bursts can be generated in the present MHD model using time series of perturbations. Furthermore, some of real radio spectra might show a superposition of the fiber bursts generated in several nearby waveguides.

Considering our simple emission model, we plan to solve the inverse problem to the presented solution: i.e., we plan to determine the density profiles of the propagating magnetoacoustic wave from the fiber burst profiles measured along the radio frequency at some specific times.

Acknowledgements. The authors thank an anonymous referee for comments that improved the paper. This research was supported by grants P209/12/0103 (GA CR), P209/10/1680 (GA CR), the research project RVO:67985815 of the Astronomical Institute AS, and the Marie Curie PIRSES-GA-2011-295272 RadioSun project. The wavelet analysis was performed with software based on tools provided by C. Torrence and G. P. Compo at http://paos. colorado. edu/research/wavelets.

\section{References}

Aschwanden, M. J. 2002, Space Sci. Rev., 101, 188

Aschwanden, M. J. 2004, Physics of Solar Corona (Germany Berlin: Springer-Verlag), 202

Aurass, H., Chernov, G. P., Karlický, M., Kurths, J., \& Mann, G. 1987, Sol. Phys., 112, 347

Bárta, M., Buechner, J., Karlický, M., \& Skála, J. 2011a, ApJ, 737, 24

Bárta, M., Buechner, J., Karlický, M., \& Kotrč, P. 2011b, ApJ, 730, 47

Benz, A. O., \& Mann, G. 1998, A\&A, 333, 1034

Bernold, T. E. X., \& Treumann, R. 1983, A\&A, 264, 677
Cargill, P. J., Vlahos, L., Baumann, G., Drake, J. F., \& Nordlund, A. 2012, Space Sci. Rev., 173, 223

Chernov, G. P. 1976, Sov. Astron., 20, 449

Chung, T. J. 2002, Computational Fluid Dynamics (New York, USA: Cambridge University Press)

Farge, M. 1992, Annu. Rev. Fluid Mech., 24, 395

Fryxell, B., Olson, K., Ricker, P., et al. 2000, ApJSS, 131, 273

Galsgaard, K., \& Roussev, I. 2002, A\&A, 383, 685

Jelínek, P., \& Karlický, M. 2012, A\&A, 537, A46

Jelínek, P., Karlický, M., \& Murawski, K. 2012, A\&A, 546, A49

Jiřička, K., Karlický, M., Kepka, O., \& Tlamicha, A. 1993, Sol. Phys., 147, 203

Jiřička, K., Karlický, M., Mészárosová, H., \& Snížek, V. 2001, A\&A, 375, 243

Karlický, M., Jelínek, P., \& Mészárosová, H. 2011, A\&A, 529, A96

Karlický, M., Bárta, M., \& Nickeler, D. 2012, A\&A, 541, A86

Kuijpers, J. 1975, Sol. Phys., 44, 143

Kuznetsov, A. A. 2006, Sol. Phys., 237, 153

Lee, D., \& Deane, A. E. 2009, J. Comput. Phys., 228, 952

Lin, J., Ko, Y.-K., Sui, L., et al. 2005, ApJ, 622, 1251

Mann, G., Karlický, M., \& Moschmann, U. 1987, Sol. Phys., 110, 381

Mészárosová, H., Karlický, M., Rybák, J., \& Jiřrička, K. 2009a, ApJ, 697, L108

Mészárosová, H., Karlický, M., Rybák, J., \& Jiřička, K. 2009b, A\&A, 502, L13

Mészárosová, H., Karlický, M., \& Rybák, J. 2011, Sol. Phys., 273, 393

Murawski, K. 2002, Analytical and Numerical Methods for Wave Propagation in Fluid Media (Singapore: World Scientific)

Nakariakov, V. M., Arber, T. D., Ault, C. E., et al. 2004, MNRAS, 349, 705

Rausche, G., Aurass, H., Mann, G., Karlický, M., \& Vocks, C. 2007, Sol. Phys., 245,327

Roberts, B., Edwin, P. M., \& Benz, A. O. 1983, Nature, 305, 688

Roberts, B., Edwin, P. M., \& Benz, A. O. 1984, ApJ, 279, 865

Torrence, C., \& Compo, G. P. 1998, Bull. Am. Meteor. Soc., 79, 61

Treumann, R. A., Güdel, M., \& Benz, A. O. 1990, A\&A, 236, 242

Winglee, R. M., \& Dulk, G. A. 1986, ApJ, 307, 808

Yasnov, L. V., \& Karlický, M. 2004, Sol. Phys., 219, 289 